\title{
Application of deuterated THENA for assigning the absolute configuration of chiral secondary alcohols
}

\author{
Soponpong, Jakapun ; Dolsophon, Kulvadee ; Thongpanchang, Chawanee ; Linden, Anthony ;
}

Thongpanchang, Tienthong

\begin{abstract}
The structure of a constrained bicyclic CDA, 1,2,3,4-tetrahydro-1,4-epoxynaphthalene-1-carboxylic acid, THENA 1, was modified by replacing both exo-methylene protons with deuterium atoms. The modified CDA, THENA-d2 2, was successfully prepared and could be used to assign the absolute configuration of chiral secondary alcohols with good reliability. Compared with THENA, the multiplicity of the methylene proton signals in the $1 \mathrm{H}$ NMR spectra of THENA-d2 derivatives is less complicated and the new CDA thus offers simpler NMR spectra for data interpretation.
\end{abstract}

DOI: https://doi.org/10.1016/j.tetlet.2019.01.013

Posted at the Zurich Open Repository and Archive, University of Zurich ZORA URL: https://doi.org/10.5167/uzh-162388

Journal Article

Accepted Version

Originally published at:

Soponpong, Jakapun; Dolsophon, Kulvadee; Thongpanchang, Chawanee; Linden, Anthony; Thongpanchang, Tienthong (2019). Application of deuterated THENA for assigning the absolute configuration of chiral secondary alcohols. Tetrahedron Letters, 60(6):497-500.

DOI: https://doi.org/10.1016/j.tetlet.2019.01.013 


\title{
On the application of deuterated THENA for assigning the absolute configuration of chiral secondary alcohols
}

\author{
Jakapun Soponpong, ${ }^{\mathrm{a}}$ Kulvadee Dolsophon, ${ }^{\mathrm{b}}$ Chawanee Thongpanchang, ${ }^{\mathrm{c}}$ Anthony Linden, ${ }^{\mathrm{d}}$ and \\ Tienthong Thongpanchang ${ }^{\mathrm{a}, \mathrm{c}}$, * \\ ${ }^{a}$ Department of Chemistry and Center of Excellence for Innovation in Chemistry (PERCH-CIC), Faculty of Science, Mahidol University, Rama 6 Road, Bangkok \\ 10400, Thailand \\ ${ }^{b}$ Department of Chemistry, Faculty of Science, Srinakharinwirot University, 114 Sukhumvit 23, Bangkok 10110, Thailand \\ ${ }^{c}$ National Center for Genetic Engineering and Biotechnology, 113 Thailand Science Park, Khlong Luang, Pathum Thani 12120, Thailand \\ ${ }^{d}$ Department of Chemistry, University of Zurich, Winterthurerstrasse 190, CH-8057 Zurich, Switzerland
}

\section{ARTICLE INFO}

Article history:

Received

Received in revised form

Accepted

Available online

Keywords:

Constrained bicyclic

Chiral derivatizing agent

Diels Alder reaction

Deuterated molecule

\section{ABSTRACT}

The structure of a constrained bicyclic CDA, 1,2,3,4-tetrahydro-1,4-epoxynaphthalene-1carboxylic acid, THENA 1, was modified by replacing both exo-methylene protons with deuterium atoms. The modified CDA, THENA- $d_{2} 2$, was successfully prepared and could be used to assign the absolute configuration of chiral secondary alcohols with good reliability. Compared with THENA, the multiplicity of the methylene proton signals in the ${ }^{1} \mathrm{H}$ NMR spectra of THENA$d_{2}$ derivatives is less complicated and the new CDA thus offers simpler NMR spectra for data interpretation.

2017 Elsevier Ltd. All rights reserved.

\section{Introduction}

The determination of the absolute configuration of chiral compounds is always a challenging question for structural chemists. With the development of high field NMR, derivatization of chiral secondary alcohols with both enantiomers of chiral derivatizing agents (CDAs), ${ }^{1}$ such as Mosher's acid (MTPA), ${ }^{2}$ has widely been used as a method to identify the absolute configuration of the alcohol of interest. However, with certain limitations of each CDA, a variety of CDAs have continuously been developed. ${ }^{3}$

In principle, the differentiation of ${ }^{1} \mathrm{H}$ NMR signals in both diastereomeric MTPA esters results from the anisotropic effect of an aromatic group and the chemical shift difference values $(\Delta \delta)$ can be used for assigning the absolute configuration. However, due to the rotation of an anisotropic group in certain CDA designs, ${ }^{4}$ each conformer can contribute to the anisotropic influence on the proton of interest and therefore cause small $\Delta \delta$. Later, 1,2,3,4tetrahydro-1,4-epoxynaphthalene-1-carboxylic acid, THENA 1, (Figure 1) was introduced as a CDA with restricted rotation of the anisotropic group caused by the bicyclic core structure. ${ }^{5}$ Although THENA is used successfully as a CDA, its four methylene protons may pose difficulty in interpreting the ${ }^{1} \mathrm{H}$ NMR spectrum of the THENA ester, especially in the high field region. In this work, we present a means of reducing the complexity of the ${ }^{1} \mathrm{H}$ NMR spectrum of THENA itself without sacrificing the simplicity and efficiency of its synthetic utility. THENA- $d_{2} 2$, in which the exo protons on the methylene carbon atoms have been replaced by deuterons, is proposed and tested with natural chiral secondary alcohols to prove its efficiency as a CDA.

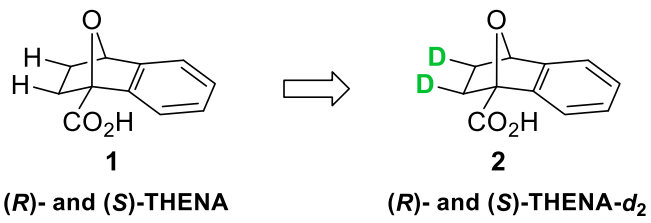

Figure1. The structures of THENA and THENA- $d_{2}$.

\section{Results and discussion}

The preparation of enantiopure THENA- $d_{2} \mathbf{2}$ followed the synthesis of THENA 1, ${ }^{5 a}$ as shown in Scheme 1. A Diels-Alder reaction between methyl furan-2-carboxylate $\mathbf{3}$ and benzyne, produced by the decomposition of diazonium salt $\mathbf{4}$ at high temperature, in refluxing 1,2-dichloroethane formed the racemic mixture of bicyclic compound ( \pm )-5 in $88 \%$ yield. Subsequent, deuteration of compound $( \pm)-5$ was affected by using a H-Cube ${ }^{\circledR}$ continuous-flow hydrogenation reactor, ${ }^{6}$ with $\mathrm{Pd} / \mathrm{C}$ catalyst and $99 \% \mathrm{D}_{2} \mathrm{O}$ as the deuterium source to afford compound $( \pm)-6$ in $99 \%$ yield $\left(99 \%\right.$ atom $\left.\mathrm{D}^{7}\right){ }^{8}$ Hydrolysis of ester $( \pm)-6$ gave acid 
( \pm )-2 (80\% yield), which was transformed to the corresponding acid chloride with oxalyl chloride and then reacted with $L$ phenylalaninol to form an amide. ${ }^{9}$ The separation of amide diastereomers was done by column chromatography to provide pure diastereomeric compounds $(\boldsymbol{R}, \boldsymbol{S})-\mathbf{7} \mathbf{a}^{10}$ and $(\boldsymbol{S}, \boldsymbol{S})-\mathbf{7} \mathbf{b}^{11}$ in $31 \%$ and $30 \%$ yield, respectively. Later, each diastereomer was hydrolyzed to finally obtain optically active THENA- $d_{2}(\boldsymbol{R})-(-)-2$ $(90 \%)$ and $(S)-(+)-2(98 \%)$.
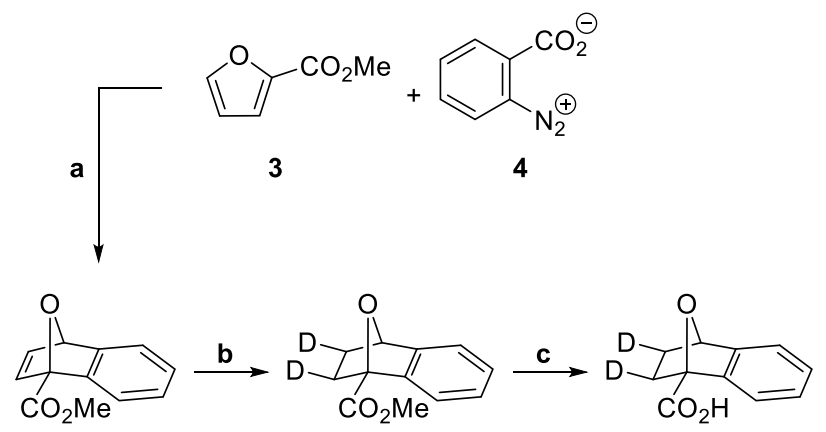

$( \pm)-5$

$( \pm)-6$

$( \pm)-2$

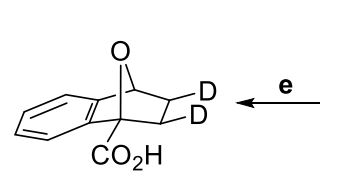

(R)-(-)-2

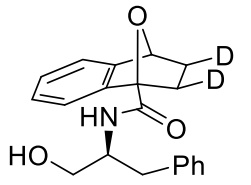

$(R, S)-7$ a<smiles>[2H]C1C2OC(c3ccccc32)C1(CO)C(=O)O</smiles>

(S)-(+)-2

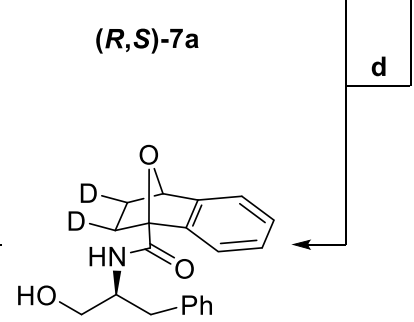

$(S, S)-7 b$

Scheme 1. Preparation of optically active THENA- $d_{2}(\boldsymbol{R})-(-)-2$ and $(S)-(+)-2^{\mathrm{a}}$

aReagents and conditions: (a) 1,2-dichloroethane, reflux, overnight, $88 \%$; (b) $\mathrm{D}_{2}, \mathrm{Pd} / \mathrm{C}, \mathrm{CH}_{2} \mathrm{Cl}_{2}, 30^{\circ} \mathrm{C}, 99 \%$; (c) $\mathrm{KOH}$ (5 eq), $\mathrm{MeOH}: 1,4-$ dioxane (1:1), rt, overnight, $80 \%$; (d) (i) $(\mathrm{COCl})_{2}$, DMF (cat.), $\mathrm{CH}_{2} \mathrm{Cl}_{2}, 0{ }^{\circ} \mathrm{C}$ to rt, $3 \mathrm{hr}$; (ii) $L$-phenylalaninol, NEt3, DMAP (cat.), $\mathrm{CH}_{2} \mathrm{Cl}_{2}$, rt, overnight, $(\boldsymbol{R}, \boldsymbol{S})-\mathbf{7} \mathbf{a}(31 \%)$ and $(\boldsymbol{S}, \boldsymbol{S})-\mathbf{7 b}(30 \%)$; (e) $\mathrm{KOH}$ (30 eq), $\mathrm{MeOH}: 1,4$-dioxane (1:1), reflux, overnight, $90 \%$ and $98 \%$ for $(R)-(-)-2$ and $(S)-(+)-2$, respectively.

Compound $(S, S)-7 b$ provided a good single crystal suitable for $\mathrm{X}$-ray diffraction analysis. ${ }^{12}$ The asymmetric unit contains two molecules of the same enantiomer with very similar conformations and slight disorder of the benzyl entity. As shown in Figure 2, the alignment of the amide $\mathrm{C}=\mathrm{O}$ and the $\mathrm{C}^{\alpha}-\mathrm{O}$ is anti-periplanar. ${ }^{13}$ Although the absolute configuration of the molecule could not be confirmed independently by the diffraction experiment, the known configuration of the $L-(S)$-phenylalaninol used as a resolving agent could be applied in constructing the model for the crystal structure refinement, from which it was possible to confirm the absolute configuration of THENA- $d_{2}$ as the $(S)$-isomer. It should be noted that the position of deuterium cannot be confirmed by X-ray crystallography. However, the syn hydrogenation at the exo position of the bridge of the [2.2.1] alkene was precedented ${ }^{14}$ and the exo-deuteration could be confirmed, based on the disappearance of the $J$-coupling between exo proton $\mathrm{H}-3$ and the bridgehead proton $\mathrm{H}-4{ }^{15}$

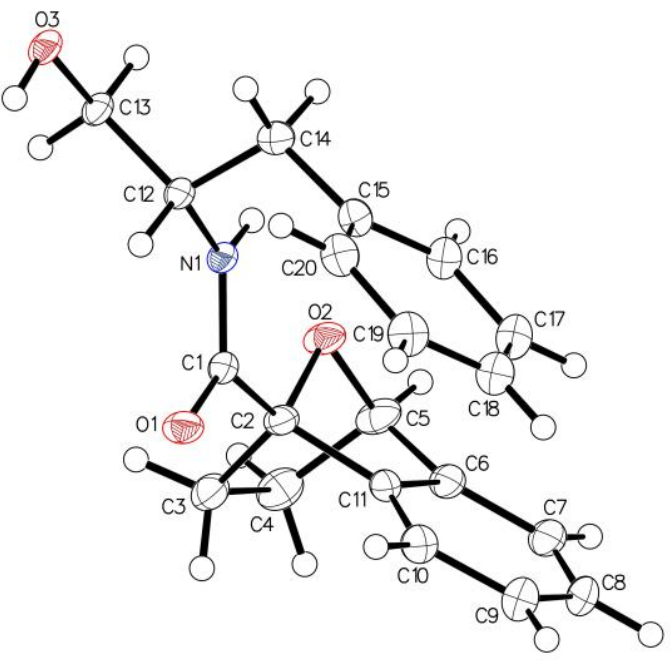

Figure 2. ORTEP diagram of the major disorder conformation of one of the two symmetry-independent molecules in the crystal structure of $(\boldsymbol{S}, \boldsymbol{S})-\mathbf{7 b}$ (drawn with $30 \%$ probability displacement ellipsoids).

To determine the absolute configuration of a chiral secondary alcohol of interest, it was first coupled with THENA- $d_{2}$ to form a pair of diastereomers. As shown in Figure 3, the array of $\mathrm{O}-\mathrm{C}^{\alpha}-\mathrm{CO}-\mathrm{O}-\mathrm{C}^{*}-\mathrm{H}$ bonds of the THENA- $d_{2}$ ester was assigned to be in a plane, called the THENA plane. ${ }^{5 a}$ Accordingly, the ${ }^{1} \mathrm{H}$ NMR chemical shifts of the protons in the alcohol substituent on the same side of this plane as the rigid aromatic group of the THENA- $d_{2}$ entity will shift to a lower field, because of the deshielding anisotropic effect. In contrast, the protons of the alcohol substituent on the other side of the THENA plane are further away from the aromatic ring, and their chemical shifts will be influenced less. Thus, the protons on substituent $\mathrm{L}_{2}$ in the $(S, ?)$ THENA- $d_{2}$ ester (Figure $3 \mathrm{~b}$ ) and on $\mathrm{L}_{1}$ in the $(R, ?)$ THENA- $d_{2}$ ester (Figure 3c) are deshielded to different extents, which results in negative and positive signs for the chemical shift difference values $\left(\Delta \delta^{S R} ; \Delta \delta^{S R}=\delta_{(S, 3)}-\delta_{(R, 3)}\right)$ for protons on the substituents $\mathrm{L}_{1}$ and $\mathrm{L}_{2}$, respectively (Figure $3 \mathrm{~d}$ ).

In accordance with the above concept, THENA- $d_{2}(\boldsymbol{R})-(-)-2$ and $(S)-(+)-2$ were activated with oxalyl chloride before being treated with a variety of optically active secondary alcohols to generate a diastereomeric pair of $(R, ?)$ - and $(S, ?)$-THENA- $d_{2}$ esters 8-27 in moderate yields. The chemical shifts of the protons in the alcohol substituent of each corresponding diastereomer were used to calculate the chemical shift difference values $\left(\Delta \delta^{S R}\right)$. If the signs of the $\Delta \delta^{S R}$ values for most of the protons on one side of the alcohol substituent (as separated by THENA plane) are negative, this group will be assigned as substituent $\mathrm{L}_{1}$ in the model (Figure $3 d)$. On the other hand, the other side of the alcohol substituent, where the $\Delta \delta^{S R}$ values for most of the protons are positive, should be specified as substituent $\mathrm{L}_{2}$. Overall, the absolute configurations determined by the THENA- $d_{2}$ method were found to be identical with the known configurations of the chiral secondary alcohols, 8 27, used for testing.

Not only can THENA- $d_{2}$ be used in the assignment of the absolute configuration of chiral secondary alcohols with satisfactory success, the high field region in the ${ }^{1} \mathrm{H}$ NMR spectra of these compounds is less complicated. For example, comparison of the high field regions in the ${ }^{1} \mathrm{H}$ NMR spectra of the $(1 R, 2 S, 5 R)$ $(-)$-menthol esters of $(S)$-THENA ${ }^{5 a}$ and $(S)$-THENA- $d_{2} 15$ illustrates clearly the decrease in the multiplicity of the methylene signals from 3 multiplet signals (the arrows in Figure 5a) to 2 doublet signals (the arrows in Figure 5b). 


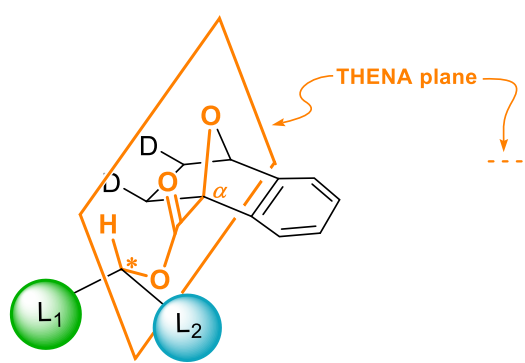

(a)

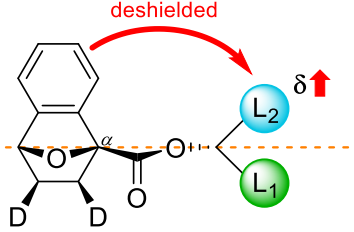

$(S, ?)$

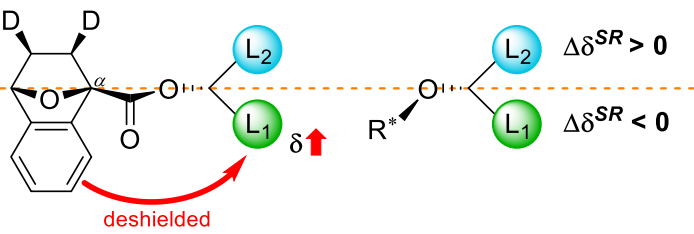

$(R, ?)$
$\Delta \delta^{S R}=\delta_{(S, \lambda)}-\delta_{(R, \lambda)}$

(d)

Figure 3. (a) The THENA plane in the structure of the ( $S$, ?)-THENA- $\mathrm{d}_{2}$ ester. (b), (c) The influence of the anisotropic group on the substituents $\mathrm{L}_{2}$ and $\mathrm{L}_{1}$ of $(S, ?)$ - and $(R, ?)$-THENA- $d_{2}$ esters, respectively. (d) The model for assigning the absolute configuration (?) of the chiral secondary alcohol by the sign of the $\Delta \delta^{S R}$ value.

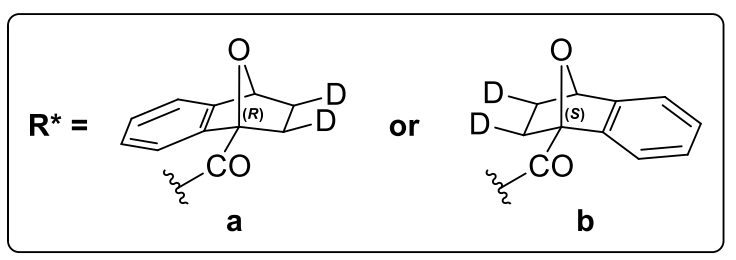

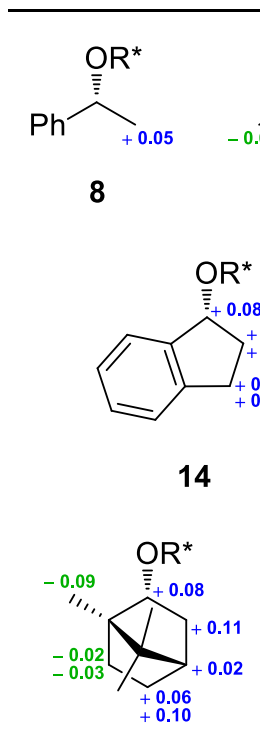

18

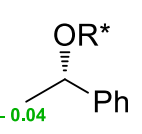

9

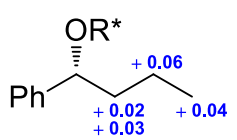

10

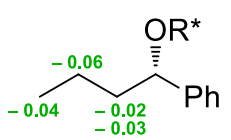

11<smiles>CC[C@H](C)O[Na]</smiles>

12<smiles>CCCCC(C)C</smiles>

13<smiles>[R7]OC1[C@H](C)CC[C@@H](C)[C@H]1C(C)C</smiles>

15

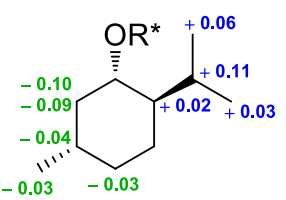

16<smiles>[R7]C1[C@H](O)[C@@H](O)CC[C@H]1C(C)C</smiles>

17

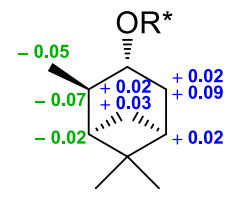

19

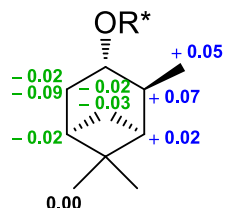

20

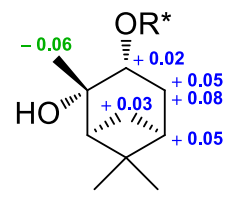

21

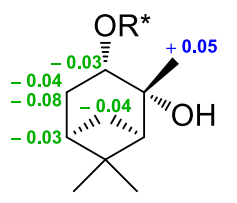

22<smiles>[R7]O[C@H]1[C@H](c2ccccc2)O[C@H]2C=CC(=O)O[C@]21[Hg]</smiles>

23

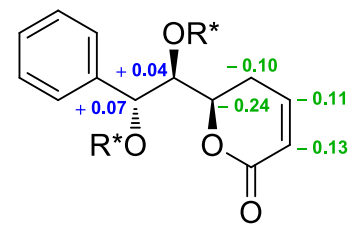

24

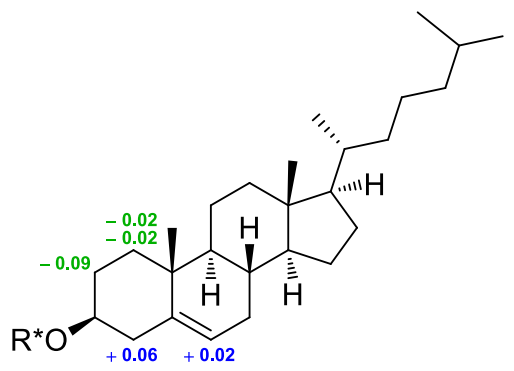

26

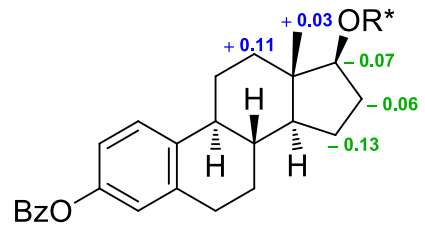

25

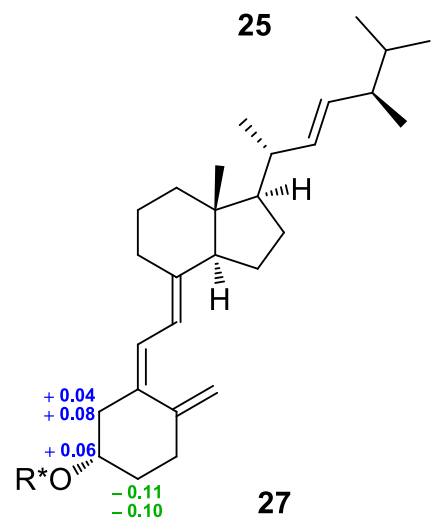

Figure 4. The $\Delta \delta^{S R}$ values of the THENA- $d_{2}$ esters of alcohols 8-27. The value less than $\pm 0.01 \mathrm{ppm}$ is omitted. 
(a)

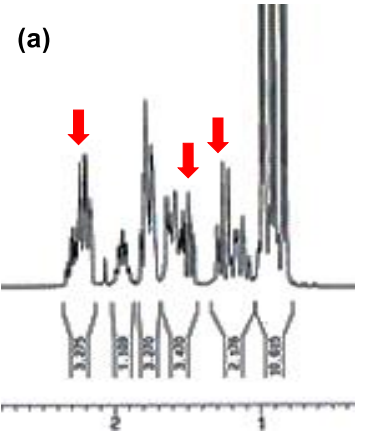

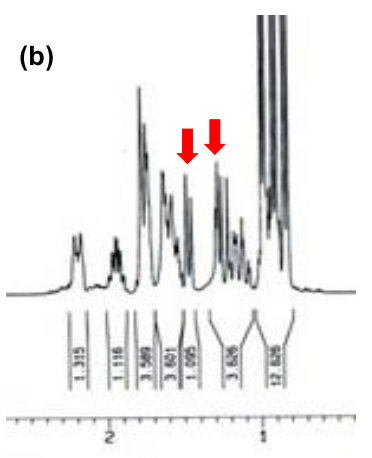

Figure 5. The ${ }^{1} \mathrm{H}$ NMR spectra in high field region of the (a) $(S)$ THENA and (b) $(S)$-THENA- $d_{2}$ esters of $(1 R, 2 S, 5 R)-(-)$-menthol 15.

\section{Conclusion}

Optically active (R)-(-)-THENA- $d_{2}$ and $(\boldsymbol{S})$-(+)-THENA- $\boldsymbol{d}_{2}$ were successfully prepared in high yield. Similar to THENA, THENA- $d_{2}$ can be used to determine the absolute configuration of chiral secondary alcohols with good reliability. Incorporation of deuterium in THENA- $d_{2}$ decreased the complexity of the ${ }^{1} \mathrm{H}$ NMR spectra. These results demonstrate the increased utility that can be afforded by constrained bicyclic CDAs when used as a means to establish the absolute configuration of compounds with more complicated structures.

\section{Acknowledgments}

Financial support and fellowships from the Faculty of Science, Mahidol University, the Thailand Research Fund (grant BRG5480020), the Development of Pharmaceuticals from Bioresources and Its Management (NRU) and the Center of Excellence for Innovation in Chemistry (PERCH-CIC) are gratefully acknowledged. We also thank Prof. Dr. Patoomratana Tuchinda and Sariyarach Thanasansurapong for pure optically active secondary alcohols $\mathbf{2 3}$ and $\mathbf{2 4}$.

\section{Supplementary Material}

The experimental procedures for preparing all compounds together with their ${ }^{1} \mathrm{H}$ and ${ }^{13} \mathrm{C}$ NMR spectra and HPLC-MS spectra can be found (in the online version) at doi:xx.xxxx/j.tetlet.xxxx.xx.xxx.

\section{References and notes}

1. (a) Seco, J. M.; Quiñoá, E.; Riguera, R. Chem. Rev. 2004, 104, 17-117. (b) Seco, J. M.; Quiñoá, E.; Riguera, R. Tetrahedron: Asymmetry 2001, 12, 2915-2925.

2. Dale, J. A.; Mosher, H. S. J. Am. Chem. Soc. 1973, 95, 512-519.

3. (a) Kuwahara, S.; Naito, J.; Yamamoto, Y.; Kasai, Y.; Fujita, T.; Noro, K.; Shimanuki, K.; Akagi, M.; Watanabe, M.; Matsumoto, T.; Watanabe, M.; Ichikawa, A.; Harada, N. Eur. J. Org. Chem. 2007, 1827-1840. (b) Kasai, Y.; Taji, H.; Fujita, T.; Yamamoto, Y.; Akagi, M.; Sugio, A.; Kuwahara, S.; Watanabe, M.; Harada, N.; Ichikawa, A.; Schurig, V. Chirality 2004, 16, 569-585. (c) Seco, J. M.; Quiñoá, E.; Riguera, R. Tetrahedron 1999, 55, 569-584. (d) Takeuchi, Y.; Fujisawa, H.; Noyori, R. Org. Lett. 2004, 6, 4607-4610. (e) PérezEstrada, S.; Joseph-Nathan, P.; Jiménez-Vázquez, H. A.; MedinaLópez, M. E.; Ayala-Mata, F.; Zepeda, L. G. J. Org. Chem. 2012, 77, $1640-1652$.

4. Latypov, S. K.; Seco, J. M.; Quiñoá, E.; Riguera, R. J. Org. Chem. 1996, $61,8569-8577$.

5. (a) Sungsuwan, S.; Ruangsupapichart, N.; Prabpai, S.; Kongsaeree, P.; Thongpanchang, T. Tetrahedron Lett. 2010, 51, 4965-4967. (b) Kornsakulkarn, J.; Dolsophon, K.; Boonyuen, N.; Boonruangprapa, T.; Rachtawee, P.; Prabpai, S.; Kongsaeree, P.; Thongpanchang, C. Tetrahedron 2011, 67, 7540-7547. (c) Daengrot, C.; Rukachaisirikul, V.; Tansakul, C.; Thongpanchang, T.; Phongpaichit, S.; Bowornwiriyapan, K.; Sakayaroj, J. J. Nat. Prod. 2015, 78, 615-622. (d) Dolsophon, K.; Ruangsupapichat, N.; Soponpong, J.; Sungsuwan, S.; Prabpai, S.; Kongsaeree, P.; Thongpanchang, T. Tetrahedron: Asymmetry 2016, 27, 1113-1120.

6. Sándor, Ö.; Chi-Ting, H.; Yang-Chang, W.; Jih-Heng, L.; Fang-Rong, C.; Ferenc, F. Molecules 2016, 21, 318.

7. The $\%$ atom $\mathrm{D}$ of compound $( \pm)-\mathbf{6}$ was calculated from the intensity of its $\left[\mathrm{M}+\mathrm{Na}^{+}\right]$signal and the intensity of the $\left[\mathrm{M}+\mathrm{Na}^{+}\right]$signal of THENA methyl ester in the HRMS spectrum of compound $( \pm)-6$

8. It should be noted that conventional deuteration with $\mathrm{D}_{2}$ on $\mathrm{Pd} / \mathrm{C}$ also gave the deuterated product in excellent yield.

9. Arita, S.; Yabuuchi, T.; Kusumi, T. Chirality 2003, 15, 609-614.

10. Diastereomeric purity of compound $(\boldsymbol{R}, \boldsymbol{S})-\mathbf{7 a}$ is $>99 \%$ de, which was calculated from a comparison of the intensity of its signal with that of the other diastereomer in the HPLC spectrum of compound $(\boldsymbol{R}, \boldsymbol{S})-7 \mathbf{a}$.

11. Diastereomeric purity of compound $(\boldsymbol{S}, \boldsymbol{S})-\mathbf{7 b}$ is $>99 \%$ de, which was calculated from a comparison of the intensity of its signal with that of the other diastereomer in the HPLC spectrum of compound $(\boldsymbol{S}, \boldsymbol{S})-\mathbf{7} \mathbf{b}$.

12. CCDC-1841008 contains the supplementary crystallographic data for this paper. The data can be obtained free of charge from The Cambridge Crystallographic Data Centre via www.ccdc.cam.ac.uk/structures.

13. Trost, B. M.; Bunt, R. C.; Pulley, S. R. J. Org. Chem. 1994, 59, 4202-4205.

14. (a) Smith, J. G.; Dibble, P. W. J. Org. Chem. 1988, 53, 1841-1848. (b) Smith, J. G.; Welankiwar, S. S.; Shantz, B. S.; Lai, E. H.; Chu, N. G. J. Org. Chem. 1980, 45, 1817-1824.

15. (a) Cokol, N. K.; Kaya, S.; Balci, M. Tetrahedron Lett. 2017, 58, 2732-2735. (b) Rowley, C. N.; Woo, T. K.; Mosey, N. J. J. Chem. Educ. 2009, 86, 199-201. 